\title{
Hollow Beam creation with continuous diffractive phase mask at PHELIX*
}

\author{
C. Brabetz ${ }^{2}$, V. Bagnoud ${ }^{1}$, S. Busold ${ }^{4}$, T. E. Cowan ${ }^{3}$, O. Deppert ${ }^{4}$, U. Eisenbarth ${ }^{1}$, S. Götte ${ }^{1}$, \\ O. Kester ${ }^{1,2}$, D. Schumacher ${ }^{1}$, B. Zielbauer ${ }^{l}$
}

${ }^{1}$ GSI Darmstadt, Germany; ${ }^{2}$ Johann Wolfgang Goethe University, Frankfurt, Germany; ${ }^{3}$ HZDR, Helmholtz center Dresden Rossendorf, Germany; ${ }^{4}$ TU Darmstadt, Darmstadt, Germany

\section{Overview}

In the framework of the Laser Ion Generation Handling and Transport (LIGHT) project, the reduction of the divergence of the laser accelerated ions is a central issue. One solution relies on engineering the electron sheath used in standard laser-driven proton acceleration (target normal sheath acceleration, TNSA) for reducing the initial divergence of the ion beam. In 2012, we conducted an experimental campaign in which "donut" focal spot have been used to drive proton acceleration. From that we have found two interesting features:

- One sees a qualitative effect of the focal spot beam shape on the ion beam divergence as expected, and

- The energy cut-off in the proton spectrum was nearly higher when a donut focus was applied, although this resulted in contradiction with the scaling law of TNSA.

\section{Report on the 2012 beamtime}

During the beamtime, we did a first run on laser-ion acceleration with engineered beams. We also focused particularly on avoiding strong wavefront distortion in the laser amplifier (astigmatism) because we saw how crucial it is for that kind of experiment. In 2012 a new PHELIX off-axis parabolic mirror was installed and produced a good hollow focus profile during alignment. However, we found that the on-shot aberrations also strongly alter the beam quality. Therefore a bending mechanism was installed to the main-mirror 1 in the main amplifier of PHELIX to correct for the thermal aberrations happening on shot and measured with a wavefront sensor. Using this pre-compensation, the alignment- beam profile looks distorted but the on-shot profile looks more promising.

\section{Results on ion acceleration with engineered la- ser beam}

In total we had 29 successful high-energy experiment shots on gold foils with different thicknesses with and without hollow beam.

In comparison to a standard Gaussian beam (blue dots in Figure 1) the focal spot diameter of the hollow beam focus increases by a factor of about 2 and therefore the peak intensity drops nearly by a factor of 4 . A troubling feature is that the maximum proton energy for the hollow beams (in red) does not depend on the laser intensity contrarily to what laser-ion acceleration scaling laws predict. For higher-order hollow beams (green dots), the focal spot was heavily distorted and resulted in a speckle-like energy distribution at low intensity. In this case a significant reduction of the proton energies was observed.

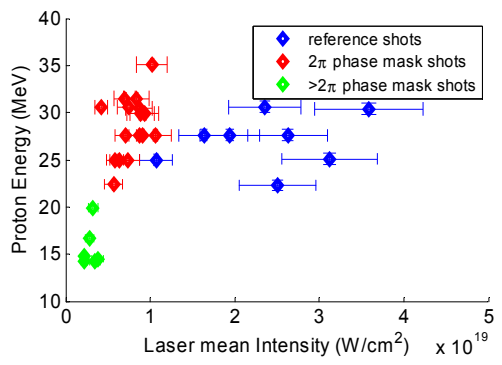

Figure 1: Proton Energy dependency on laser intensity.

From an angular distribution stand-point, the proton beams created with the hollow laser beam clearly show a systematic trend to lower divergences and higher proton yields. Taking the more aberrated shots into account we observe other effects on the ion beam. Some type of shots with a hollow beam profile broke down to 2 similar strong focal spots shows a divergence reduction in only on dimension while other shots with the hollow beam phase on thick targets shows unexpected high proton energies.

\section{$3 w$ on-shot focus diagnos- tics}

We set up a new imaging diagnostic for measuring the laser focal spot on target during the shot [1]. The idea was to look at the relativistic oscillation plasma surface that generates harmonics. Then filter for only the $3 \mathrm{w}$ light that is specular reflect-

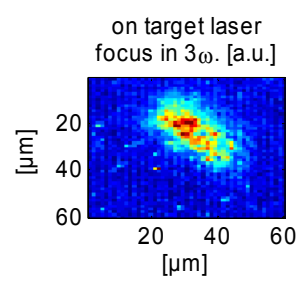
cus in $3 \mathrm{w}$.
Figure 2: on-shot foed from the target surface and is linked directly to the laser focal spot and the TNSA source size. As the components were not available in the beginning of the experiment it was just installed in the last two days. Therefore there was not enough time for optimization of the diagnostic. We know that the imaging system was strongly affected by astigmatism (duo to passing through a thick glass substrate). But still we can say that the source size was smaller than $80 \times 80 \mu \mathrm{m}^{2}$ (Figure 2).

\section{References}

[1] B. Dromey et al., Laser and Particle Beams 27, $243-$ 248 (2009).

* Work supported by HIC for FAIR. 